\title{
Aplicações isoladas ou Associadas de Diuron, Oxyfluorfen e PROMETRYNe PARA O CONTROLE DE Euphorbia heterophylla
}

\author{
Isolated or Combined Application of Diuron, Oxyfluorfen and Prometryn for Euphorbia \\ heterophylla Control
}
OLIVEIRA JR., R.S. ${ }^{2}$, CARNEIRO, J.C. ${ }^{3}$, CONSTANTIN, J. ${ }^{2}$, SANTOS, G. ${ }^{4}$, MARTINI, P.E. ${ }^{5}$, FRANCISCHINI, A.C. ${ }^{4}$ e OSIPE, J.B. ${ }^{4}$

\begin{abstract}
RESUMO - A aplicação em pré-emergência no início do ciclo do algodoeiro é prática consagrada entre os produtores. Embora os herbicidas diuron, oxyfluorfen e prometryne sejam opções para uso nessa cultura, há informações limitadas referentes à eficácia desses herbicidas no controle de Euphorbia heterophylla. O objetivo do presente trabalho foi determinar a curva de dose-resposta de diuron, oxyfluorfen e prometryne para o controle de E. heterophylla, bem como analisar a eficiência biológica de misturas desses herbicidas no manejo dessa espécie. O trabalho foi composto por seis experimentos conduzidos simultaneamente em casa de vegetação, sendo três deles com doses crescentes de cada herbicida aplicado isoladamente e três compostos por misturas dos herbicidas dois a dois. A curva de dose-resposta foi ajustada para os dados das avaliações de porcentagem de redução de massa seca aos 28 dias após a aplicação. Determinoaram-se as doses $\mathrm{I}_{80}$ e $\mathrm{I}_{95}$, que correspondem ao controle de $80 \%$ e 95\%, respectivamente, de controle visual ou redução da massa seca. Diuron, oxyfluorfen e prometryne, aplicados isoladamente, mostraram-se eficientes, dentro do intervalo de doses utilizado, no controle de E. heterophylla. As misturas contendo oxyfluorfen apresentaram-se, de modo geral, como aditivas ou antagonísticas, ao passo que misturas de diuron+prometryne foram aditivas ou sinergisticas. Das três combinações de produtos possiveis, oxyfluorfen+prometryne foi a associação que resultou em menor número de tratamentos que promoveram controle aceitável do leiteiro.
\end{abstract}

Palavras-chave: eficácia, curva de dose-resposta, leiteiro, mistura em tanque.

\begin{abstract}
Pre-emergence herbicide application at the beginning of the crop cycle is a consecrated practice among cotton farmers. Although herbicides, such as diuron, oxyfluorfen and prometryn, are options for this crop, not much is known about their effect on Euphorbia heterophylla. This work aimed to elaborate dose-response curves for diuron, oxyfluorfen and prometryn in relation to E. heterophylla control, as well as to provide biological efficiency data for tank mixtures of these herbicides. Thus, six experiments were simultaneously carried out under greenhouse conditions, with three consisting of increasing doses of each herbicide applied isolated and three of tank mixtures of two herbicides. The dose-response curve was adjusted for \% of biomass reduction at 28 days after application; $I_{80}$ and $I_{95}$ (respectively dose for $80 \%$ and $95 \%$ of weed control and biomass reduction), were also determined. Diuron, oxyfluorfen and prometryn applied alone were effective within the range of the doses evaluated to control $\boldsymbol{E}$. heterophylla. In general, tank mixtures containing oxyfluorfen were considered additive or antagonistic, while tank mixtures of diuron + prometryn were additive or synergistic. Out of the three possible combinations evaluated, oxyfluorfen + prometryn resulted in the smallest number of combinations promoting acceptable control of wild poinsettia.
\end{abstract}

Keywords: efficacy, dose-response curve, wild poinsettia, tank mixture.

Recebido para publicação em 12.4.2010 e aprovado em 9.9.2010.

2 Professor Associado, Núcleo de Estudos Avançados em Ciência das Plantas Daninhas - NAPD/UEM, Dep. de Agronomia, Universidade Estadual de Maringá - UEM, Av. Colombo 5790, 87020-9000 Maringá-PR; ${ }^{3}$ Engâa-Agrạ ., Mestre na área de Proteção de Plantas; ${ }^{4}$ Eng $^{0}$-Agr ${ }^{\circ}$., Mestrando(a) do Curso de Pós-Graduação em Agronomia na área de Proteção de Plantas, NAPD/UEM; ${ }^{5}$ Discente do curso de Agronomia, UEM. 


\section{INTRODUÇÃO}

O leiteiro ou amendoim-bravo (Euphorbia heterophylla) é uma espécie daninha da família Euphorbiaceae, cujas plantas apresentam rápido crescimento e formam uma densa cobertura vegetal, ocasionando grandes perdas em rendimento e qualidade quando em competição com espécies cultivadas. Essas perdas ocorrem devido à competição por água, luz, nutrientes, $\mathrm{CO}_{2}$ e oxigênio.

No caso da cultura do algodão, as plantas infestantes podem causar reduções de até $90,2 \%$ na produtividade, devido à interferência das plantas daninhas durante todo o ciclo da cultura (Laca-Buendia, 1990).

Além da redução na produtividade, a presença das infestantes pode reduzir a qualidade e a remuneração paga pelas fibras colhidas; assim, existe a necessidade de a colheita ser realizada no limpo.

Um dos principais métodos de controle das plantas daninhas é a utilização de herbicidas aplicados em pré-emergência no início do ciclo do algodoeiro, sendo uma prática consagrada entre os produtores de algodão. Destina-se, basicamente, ao controle dos primeiros fluxos de emergência de plantas daninhas, os quais apresentam grande importância do ponto de vista da interferência inicial.

$\mathrm{O}$ uso continuado de herbicidas com o mesmo mecanismo de ação pode contribuir para a seleção de populações de plantas daninhas resistentes ou tolerantes a esse mecanismo de ação. Uma das principais práticas de manejo para prevenir ou manejar áreas que apresentam esses problemas é a alternância de mecanismos de ação de herbicidas.

No caso de $E$. heterophylla, a reconhecida eficácia e o uso continuado de herbicidas inibidores da ALS acabaram por selecionar diversos biótipos resistentes aos principais produtos usados no seu controle, como aqueles identificados em lavouras de soja nos Estados do Rio Grande do Sul, Paraná e Mato Grosso do Sul, onde esses produtos são empregados há vários anos (Vargas et al., 1999).

Os herbicidas diuron, oxyfluorfen e prometryne apresentam-se como opções registradas para uso na cultura, porém há poucas informações disponiveis referentes à eficiência desses herbicidas no controle de E. heterophylla. Estudos têm mostrado que combinações de herbicidas podem aumentar a eficiência e ampliar o espectro de controle de plantas daninhas controladas, devido ao fato de que cada herbicida é especialmente eficiente para determinadas espécies de plantas daninhas.

A utilização de combinações pode eventualmente tornar-se mais econômica pelo uso de doses menores e pela redução do número de aplicações. Cruz \& Toledo (1982), estudando o efeito de misturas herbicidas no controle de plantas daninhas em algodoeiro em pré-emergência, constataram que, nas condições de Mogi-Mirim, no Estado de São Paulo, a mistura de alachlor+diuron $\left(3,0+1,0 \mathrm{~kg} \mathrm{ha}^{-1}\right)$, aplicada em pré-emegência, proporcionou o melhor controle do complexo de plantas daninhas por um período de 100 dias após a emergência.

A relação entre doses de herbicidas e controle das plantas é de fundamental importância para o entendimento de diversos aspectos relacionados à eficácia dos herbicidas (Seefeldt et al., 1995). Diversos autores têm utilizado e recomendado a curva de dose-resposta para estudar o efeito biológico dos herbicidas, seja para avaliar o controle e/ou resistência de plantas daninhas a herbicidas ou para verificar a persistência de herbicidas no solo (Monquero et al., 2000).

Nesse sentido, devido ao significativo aumento dos problemas relacionados ao controle ineficiente de Euphorbia heterophylla na cultura do algodoeiro e à limitada disponibilidade de herbicidas registrados para uso no início do ciclo da cultura, o objetivo deste trabalho foi determinar curvas de dose-resposta de diuron, oxyfluorfen e prometryne em relação ao controle de E. heterophylla, bem como analisar a eficiência biológica de misturas desses herbicidas no controle dessa espécie.

\section{MATERIAL E MÉTODOS}

Foram conduzidos seis experimentos em casa de vegetação, durante o período de outubro de 2007 a dezembro de 2008. Foram utilizadas amostras de solo proveniente do distrito de Iguatemi (Maringá-PR; 2321'39,63"S e $\left.52^{\circ} 03^{\prime \prime} 57,11 " \mathrm{~W}\right)$, coletadas na profundidade de 0 a $20 \mathrm{~cm}$. 
O solo da área experimental foi classificado como ARGISSOLO VERMELHO distrófico e apresentou $37 \%$ de areia grossa, $32 \%$ de areia fina, $23 \%$ de argila e $8 \%$ de silte. Em relação à análise química, apresentou $\mathrm{pH}$ de 4,5 em $\mathrm{CaCl}_{2} ; 4,96 \mathrm{cmol}_{\mathrm{c}} \mathrm{dm}^{-3}$ de $\mathrm{H}^{+}+\mathrm{Al}^{3+}$; $0,97 \mathrm{cmol}_{\mathrm{c}} \mathrm{dm}^{-3}$ de $\mathrm{Ca}^{+2} ; 0,78 \mathrm{cmol}_{\mathrm{c}} \mathrm{dm}^{-3} \mathrm{de}$ $\mathrm{Mg}^{+2} ; 0,24 \mathrm{cmol}_{\mathrm{c}} \mathrm{dm}^{-3} \mathrm{de} \mathrm{K}^{+} ; 19,2 \mathrm{mg} \mathrm{dm}^{-3}$ de P; e 13,68 $\mathrm{g} \mathrm{dm}^{-3}$ de C. Em todas as etapas deste trabalho, as unidades experimentais foram constituídas de vasos com capacidade para $3 \mathrm{dm}^{-3}$ de solo.

Para as aplicações dos herbicidas realizadas em todos os experimentos, foi utilizado pulverizador costal sob pressão constante, pressurizado a $\mathrm{CO}_{2}$ e equipado com pontas tipo leque XR-110.02, operando à pressão de $2,0 \mathrm{kgf} \mathrm{cm}^{-2}$, espaçadas de $0,5 \mathrm{~m}$ entre si e posicionadas a $0,5 \mathrm{~m}$ da superficie do alvo, proporcionando uma vazão aproximada de $200 \mathrm{~L} \mathrm{ha}^{-1}$. As condições no momento da aplicação dos tratamentos foram de velocidade do vento inferior a $5 \mathrm{~km} \mathrm{~h}^{-1}$, solo úmido, temperatura do ar de $25{ }^{\circ} \mathrm{C}$ e umidade relativa de $84 \%$.

Na primeira etapa, foram conduzidos três experimentos, um para cada herbicida (diuron, oxyfluorfen e prometryne), com a finalidade de avaliar a eficácia dos herbicidas em relação ao controle de E. heterophylla. As doses utilizadas foram de $0,0,25,0,50,1,00$ e $2,00 \mathrm{~kg}$ diuron $\mathrm{ha}^{-1} ; 0,0,048,0,096,0,192 \mathrm{e}$

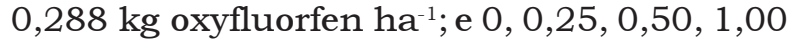
e $2,00 \mathrm{~kg}$ prometryne ha- ${ }^{-1}$. intervalo de doses foi estabelecido variando-as de zero até a dose máxima comumente utilizada para esse tipo de solo.

Após o preparo do solo, foram semeadas 30 sementes de $E$. heterophylla por vaso, na profundidade de 2 a $3 \mathrm{~cm}$. Após a semeadura, os herbicidas diuron, oxyfluorfen e prometryne foram aplicados, respectivamente, para os experimentos 1, 2 e 3 . Após a emergência das plantas, foi feito controle diário da umidade dos vasos.

$\mathrm{Na}$ segunda etapa foram conduzidos três experimentos, visando avaliar a eficácia agronômica das misturas dos herbicidas, dois a dois, seguindo-se as mesmas condições dos experimentos 1 a 3; os tratamentos de cada experimento foram constituídos pelas diferentes combinações de doses dos produtos. As doses de cada herbicida utilizadas (em $\left.\mathrm{kg} \mathrm{ha}^{-1}\right)$ foram as seguintes: experimento 4 (diuron + prometryne): $(0+0),(0,125+0,125),(0,250+$ $0,250),(0,500+0,500),(1,000+1,000),(0,250+$ $0,125), \quad(0,500+0,250), \quad(1,000+0,500)$, $(2,000+1,000),(0,125+0,250),(0,250+0,500)$, $(0,500+1,000),(1,000+2,000)$ e $(2,000+2,000)$; experimento 5 (diuron+oxyfluorfen): $(0+0)$, $(0,250+0,024),(1,000+0,096),(1,000+0,192)$, $(0,125+0,024),(0,250+0,048),(0,500+0,096)$, $(1,000+0,144),(0,125+0,048),(0,250+0,096)$, $(0,500+0,192),(1,000+0,288),(0,250+0,048)$, $(1,000+0,192)$ e $(2,000+0,288)$; e experimento 6 (prometryne + oxyfluorfen): $(0+0),(0,250+0,024)$, $(0,500+0,048),(0,550+0,096),(1,000+0,096)$, $(0,125+0,024),(1,000+0,144),(0,125+0,048)$, $(0,250+0,096), \quad(0,500+0,192), \quad(1,000+$ $0,288),(0,250+0,048),(1,000+0,192) \mathrm{e}$ $(2,000+0,288)$.

Em todos os experimentos, o controle de $E$. heterophylla foi avaliado por meio de escala visual de notas (0 - 100\%) aos 14 e 28 dias após aplicação (DAA). O valor correspondeu à ausência de controle e 100 à morte total das plantas, comparados à testemunha sem aplicação de herbicida. Na última avaliação de controle, foi realizada a coleta da parte aérea do leiteiro, para determinar o acúmulo de massa seca, com posterior secagem em estufa de ventilação forçada a $55^{\circ} \mathrm{C}$ até peso constante.

Os dados de biomassa foram corrigidos para valores percentuais, partindo-se do princípio de que os vasos que permaneceram sem aplicação de herbicida produziram $100 \%$ de biomassa, e os demais, percentuais dessa biomassa, em consequência da redução imposta pelo herbicida. Todos os experimentos foram conduzidos no delineamento de blocos casualizados com quatro repetições.

Nos três experimentos com doses crescentes dos herbicidas isolados, os resultados da avaliação visual de controle e da produção de biomassa foram inicialmente submetidos à análise de variância e, posteriormente, à análise de regressão. Em seguida, os dados foram ajustados ao modelo não linear, segundo modelo proposto por Seefeldt et al. (1995): 


$$
y=a+\frac{b}{\left[1+\left(\frac{x}{c}\right)^{d}\right]}
$$

em que: $y=$ porcentagem de controle ou de biomassa; $x=$ dose do herbicida em kg i.a. ha ${ }^{-1} ; a, b, c, d=$ coeficientes da curva, de modo que: $a=$ limite inferior da curva; $b=$ diferença entre o ponto máximo e o mínimo da curva; $c=$ dose que proporciona $50 \%$ de resposta da variável dependente; $d=$ declividade da curva ao redor de $c$.

A partir das equações log-logísticas foram elaboradas as curvas de dose-resposta. Com base nos modelos ajustados, realizou-se o cálculo da dose do herbicida, em kg i.a. ha-1, que proporcionaria 50, 80 e $95 \%$ de controle ou de redução do acúmulo de biomassa do leiteiro. Para realização do cálculo, optou-se pela inversão do modelo log-logístico, deixandoo em função de $y$. A realização deste cálculo matemático permite a correção de eventuais distorções do modelo, oferecendo valores mais próximos dos reais:

$$
x=c * \sqrt[d]{\frac{b}{(y-a)}-1}
$$

Para análise dos dados referentes às associações de herbicidas, realizou-se análise de variância, e as médias de controle dos tratamentos com herbicidas foram comparadas com a testemunha sem aplicação, por meio do teste de Dunett a 5\% de probabilidade, utilizando-se o programa estatístico SAEG 5.0. (SAEG, 1997). Como complemento do trabalho, nos experimentos que envolviam associações de herbicidas, realizou-se o cálculo matemático para verificação de possíveis efeitos entre as misturas, de acordo com modelo proposto por Colby (1967):

$$
E=x+\frac{y(100-x)}{100}
$$

em que $E$ = redução de crescimento esperado ou porcentagem de controle esperada pela mistura de herbicidas; e $x$ e $y=$ redução de crescimento proporcionada ou porcentagem de controle dos herbicidas aplicados isoladamente.
Segundo Colby (1967), quando a resposta da mistura é maior que a esperada, a mistura é sinergística; quando menor que a esperada, é antagonística; e, quando igual, aditiva.

\section{RESULTADOS E DISCUSSÃO}

\section{Eficácia no controle de $E$. heterophylla com herbicidas aplicados isoladamente}

Com a aplicação de regressões não lineares aos dados obtidos, foram definidos os parâmetros $a, b, c$ e $d$ da equação log-logística (Seefeldt et al., 1995), que estão apresentados na Tabela 1. A partir desses parâmetros, foram calculados os valores de $\mathrm{I}_{50}, \mathrm{I}_{80}$ e $\mathrm{I}_{95}$ para todas as variáveis analisadas de Euphorbia heterophylla (Tabela 2).

Os valores de produção relativa de massa seca encontram-se na Figura 1. Dentro do intervalo de doses testado, foi possivel obter $\mathrm{I}_{80}$ de cada herbicida avaliado.

\section{Controle de $E$. heterophylla com diuron}

E. heterophylla foi eficientemente controlada pelo herbicida diuron em todas as avaliações, cujos valores de controle visual encontrados para a dose máxima mantiveramse superiores a $99 \%$ (Tabela 2).

Quanto às variáveis analisadas, a dose para alcançar controle satisfatório $\left(\geq 80 \%-\mathrm{I}_{80}\right)$ de $E$. heterophylla variou entre 0,778 e $0,911 \mathrm{~kg}$ diuron $\mathrm{ha}^{-1}$, ao passo que para obter excelente controle $(\geq 95 \%)$ foram necessárias doses entre 1,030 e $1,215 \mathrm{~kg} \mathrm{ha}^{-1}$ (Tabela 2).

Dourado Neto et al. (2005) obtiveram controle de aproximadamente 95\% de $E$. heterophylla ao utilizarem doses de diuron de 1,6 (aos 10 DAA) e 2,0 kg ha ${ }^{1}$ (aos 30 DAA), respectivamente. Observa-se, nesse trabalho, que houve necessidade de doses mais altas do que as obtidas no presente trabalho. Esse fato pode estar associado ao maior teor de argila nos solos dos referidos trabalhos, visto que ambos eram de textura argilosa.

A sorção do diuron e, consequentemente, sua lixiviação são governadas principalmente pela fração orgânica do solo (Prata \& Lavorenti, 2000). A molécula é fortemente adsorvida pelos 
Tabela 1 - Estimativas dos parâmetros a, b, c e d e do coefíciente de determinação ( $\left.\mathrm{R}^{2}\right)$ do modelo log-logístico, ajustados para diuron, oxyfluorfen e prometryne, em relação à porcentagem de controle aos 14 e 28 dias após aplicação (DAA), respectivamente, e porcentagem de redução de massa seca de E. heterophylla. Maringá-PR - 2007/2009

\begin{tabular}{|c|c|c|c|c|c|}
\hline \multirow{2}{*}{ Variável } & \multicolumn{5}{|c|}{ Diuron } \\
\hline & $\mathrm{a}$ & $\mathrm{b}$ & $\mathrm{c}$ & $\mathrm{d}$ & $\mathrm{R}^{2}$ \\
\hline Controle (\%) 14 DAA & 2,8852 & 97,0167 & 0,7112 & $-5,4775$ & 0,97 \\
\hline Controle (\%) 28 DAA & 0,0276 & 100,1407 & 0,6041 & $-5,4548$ & 0,92 \\
\hline \multirow[t]{2}{*}{ Massa Seca (\%) } & 4,7749 & 95,2517 & 0,6449 & $-5,4764$ & 0,99 \\
\hline & \multicolumn{5}{|c|}{ Oxyfluorfen } \\
\hline Controle (\%) 14 DAA & 0,0255 & 120,00 & 0,1061 & $-1,4183$ & 0,89 \\
\hline Controle (\%) 28 DAA & 0,0100 & 109,3466 & 0,0559 & $-1,1996$ & 0,94 \\
\hline \multirow[t]{2}{*}{ Massa Seca (\%) } & 0,1001 & 88,1811 & 0,0739 & $-3,3135$ & 0,96 \\
\hline & \multicolumn{5}{|c|}{ Prometryne } \\
\hline Controle (\%) 14 DAA & 0,3178 & 85,2954 & 0,9312 & $-3,8218$ & 0,94 \\
\hline Controle (\%) 28 DAA & 0,0130 & 91,1335 & 0,4822 & $-3,0572$ & 0,96 \\
\hline Massa Seca (\%) & 0,1000 & 96,4588 & 0,4529 & $-2,7287$ & 0,98 \\
\hline
\end{tabular}

Tabela 2 - Valores de $\mathrm{I}_{50} \mathrm{I}_{80}$ e $\mathrm{I}_{95}$ (kg i.a. ha ${ }^{-1}$ ) e controle ou biomassa percentual (ajustado - A e observado - O) aos 14 e 28 dias após a aplicação (DAA) na dose recomendada de diuron, oxyfluorfen e prometryne ( $\left.\mathrm{kg} \mathrm{ha}^{-1}\right)$, respectivamente, para o controle de E. heterophylla. Maringá-PR - 2007/2009

\begin{tabular}{|c|c|c|c|c|c|}
\hline \multirow[t]{2}{*}{ Variável } & \multicolumn{3}{|c|}{$\begin{array}{c}\mathrm{I} \\
\left(\mathrm{kg} \mathrm{ha}^{-1}\right)\end{array}$} & \multicolumn{2}{|c|}{$\begin{array}{c}\% \text { de controle para a dose máxima utilizada } \\
\text { Diuron }\left(2 \mathrm{~kg} \mathrm{ha}^{-1}\right)\end{array}$} \\
\hline & 50 & 80 & 95 & Ajustado & Observado \\
\hline \multicolumn{6}{|c|}{ Diuron } \\
\hline Controle (\%) 14 DAA & 0,703 & 0,911 & 1,215 & 99,57 & 99,50 \\
\hline Controle (\%) 28 DAA & 0,603 & 0,778 & 1,030 & 100,02 & 100,00 \\
\hline Massa Seca (\%) & 0,633 & 0,821 & 1,093 & 99,83 & 100,00 \\
\hline \multirow[t]{2}{*}{ Variável } & \multicolumn{3}{|c|}{$\begin{array}{c}\mathrm{I} \\
\left(\mathrm{kg} \mathrm{ha}^{-1}\right)\end{array}$} & \multicolumn{2}{|c|}{ Oxyfluorfen $\left(0,288 \mathrm{~kg} \mathrm{ha}^{-1}\right)$} \\
\hline & 50 & 80 & 95 & Ajustado & Observado \\
\hline \multicolumn{6}{|c|}{ Oxyfluorfen } \\
\hline Controle (\%) 14 DAA & 0,083 & 0,173 & 0,272 & 96,61 & 98,50 \\
\hline Controle (\%) 28 DAA & 0,048 & 0,129 & 0,270 & 95,92 & 99,00 \\
\hline Massa Seca (\%) & 0,080 & 0,147 & $>0,288$ & 87,32 & 96,74 \\
\hline \multirow[t]{2}{*}{ Variável } & \multicolumn{3}{|c|}{$\begin{array}{c}\mathrm{I} \\
\left(\mathrm{kg} \mathrm{ha}^{-1}\right)\end{array}$} & \multicolumn{2}{|c|}{ Prometryne $\left(2 \mathrm{~kg} \mathrm{ha}^{-1}\right)$} \\
\hline & 50 & 80 & 95 & Ajustado & Observado \\
\hline \multicolumn{6}{|c|}{ Prometryne } \\
\hline Controle (\%) 14 DAA & 1,016 & 1,864 & $>2,00$ & 81,25 & 81,25 \\
\hline Controle (\%) 28 DAA & 0,513 & 0,918 & $>2,00$ & 90,10 & 95,75 \\
\hline Massa Seca (\%) & 0,465 & 0,806 & $>2,00$ & 94,91 & 99,13 \\
\hline
\end{tabular}

Obs.: sinal > significa a necessidade de doses maiores do que as utilizadas neste estudo.

coloides de argila ou matéria orgânica e, por essa razão, a dose adequada é altamente dependente das características do solo (Rodrigues \& Almeida, 2005). No entanto, em solos que apresentam baixo teor de argila e matéria orgânica, como o utilizado neste trabalho, pode ocorrer lixiviação moderada. Esse fato pode inclusive tornar o herbicida 

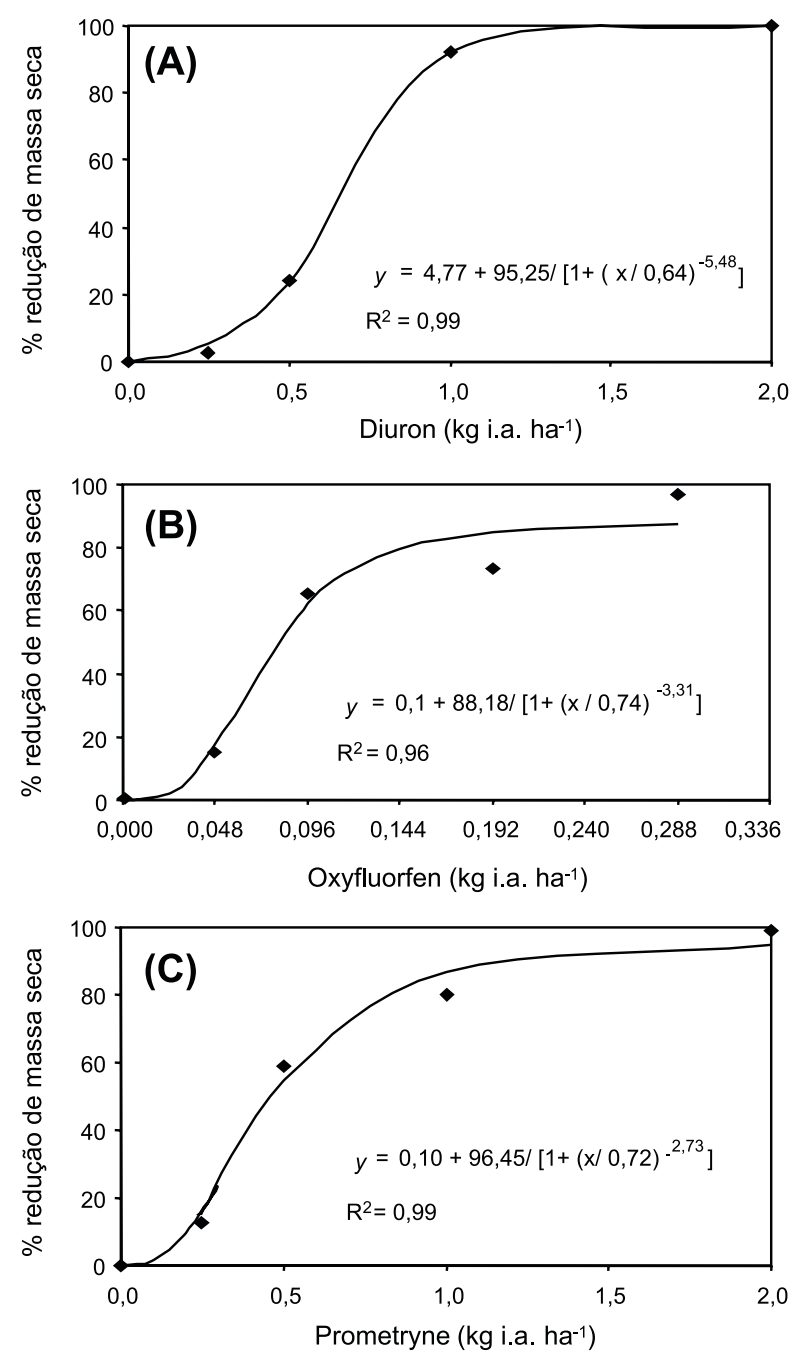

Figura 1 - Curvas de dose-resposta de diuron (A), oxyfluorfen $(B)$ e prometryne $(C)$ em relação à porcentagem de redução de massa seca da parte aérea de E. heterophylla.

mais eficiente, movendo-o da superficie do solo para onde estão concentradas as sementes de plantas daninhas (Oliveira, 2001).

Dessa forma, pode-se supor que o baixo teor de matéria orgânica desse solo propiciou maior disponibilidade do produto na solução do solo, o que contribuiu para obtenção de controles satisfatórios de E. heterophylla.

\section{Controle de E. heterophylla com oxyfluorfen}

O oxyfluorfen mostrou-se eficiente no controle de E. heterophylla. Em todas as avaliações, os valores de controle visual observados para a dose máxima mantiveram-se superiores a 95\% (Tabela 2).

Em todas as variáveis-resposta dessa espécie, observaram-se controles de $80 \%$ com doses variando entre 0,129 e $0,172 \mathrm{~kg} \mathrm{ha}^{-1}$ (Tabela 2). Para obtenção de controle excelente ( $\geq 95 \%$ ), o leiteiro exigiu doses entre 0,270 e $0,272 \mathrm{~kg} \mathrm{ha}^{-1}$. No entanto, para redução de $95 \%$ da massa seca da parte aérea, essa espécie exigiu doses maiores do que as utilizadas neste estudo $\left(>0,288 \mathrm{~kg} \mathrm{ha}^{-1}\right)$ (Tabela 2).

Estudos realizados por Castro et al. (2002) na cultura do girassol, em solo com $77,5 \%$ de argila e 19,8 $\mathrm{g} \mathrm{dm}^{-3}$ de C, demonstraram que, em campo, a dose de $0,36 \mathrm{~kg}$ oxyfluorfen $\mathrm{ha}^{-1}$ foi eficiente no controle de E. heterophylla, apresentando 97 e $95 \%$ de controle aos 25 e 35 DAA, respectivamente. Observa-se, portanto, que foram necessárias doses mais altas para o controle de E. heterophylla do que as avaliadas neste trabalho. Comparando os dois solos, observa-se também que no trabalho de Castro et al. (2002) o solo apresentava teor de argila quatro vezes maior e $5,7 \mathrm{~g} \mathrm{dm}^{-3}$ mais $\mathrm{C}$ orgânico do que o solo utilizado neste experimento.

Portanto, o solo em que foi realizado este trabalho pode ter influenciado na maior disponibilidade de oxyfluorfen e, consequentemente, na elevada eficiência do herbicida até 28 DAA.

\section{Controle de E. heterophylla com prometryne}

A dose máxima de prometryne proporcionou controle superior a $81 \%$. Para todas as variáveis analisadas neste estudo, controle considerado satisfatório $(80 \%)$ de $E$. heterophylla foi alcançado por doses que variaram de 0,806 a $1,864 \mathrm{~kg} \mathrm{ha}^{-1}$. Para controle de $95 \%\left(I_{95}\right)$, essa espécie exigiu dose maior do que a máxima testada neste estudo $\left(>2,00 \mathrm{~kg} \mathrm{ha}^{-1}\right)$.

Monquero \& Silva (2007), avaliando a eficácia agronômica de sulfosate $(0,66$ e $\left.1,32 \mathrm{~kg} \mathrm{ha}^{-1}\right)$ e glyphosate $\left(0,72 \mathrm{e} 1,44 \mathrm{~kg} \mathrm{ha}^{-1}\right)$ no controle de E. heterophylla e Ipomoea pupurea, concluíram que E. heterophylla não foi eficientemente controlada; a maior dose de glyphosate proporcionou controle de apenas 
$60 \%$. Para o sulfosate, o controle alcançado foi de $75 \%$, evidenciando que E. heterophylla é uma espécie de difícil controle.

Quanto às doses máximas utilizadas neste experimento, foi possivel obter controle visual de 100, 99 e 95,75\% para os herbicidas diuron, oxyfluorfen e prometryne, respectivamente. Os excelentes controles observados foram proporcionados por doses que representam 100, 40 e 100\% das doses máximas de diuron, oxyfluorfen e prometryne, recomendadas para aplicação em pré-emergência nesse tipo de solo para a cultura do algodão, segundo Rodrigues \& Almeida (1998).

\section{Eficácia no controle de $E$. heterophylla com associação de herbicidas}

\section{Diuron+prometryne}

Na Tabela 3 são apresentados os valores de massa seca e porcentagem de controle visual aos 14, 21 e 28 DAA. Para os herbicidas utilizados em mistura, pode-se observar que a massa seca obtida para as doses de $1,000+$ $1,000,1,000+0,500,2,000+1,000,1,000+2,000$ e 2,000+2,000 $\mathrm{kg} \mathrm{ha}^{-1}$ foi significativamente inferior à da testemunha. Em suma, combinações desses herbicidas em doses a partir de $1,0 \mathrm{~kg} \mathrm{ha}^{-1}$ de diuron e $0,500 \mathrm{~kg} \mathrm{ha}^{-1} \mathrm{de}$ prometryne promoveram efetiva redução do acúmulo de massa seca do leiteiro.

Em relação à porcentagem de controle visual (Tabela 3), excelente controle aos 28 DAA (> 95\%) foi obtido pelas misturas de $1,000+1,000,1,000+0,500,2,000+1,000$, $1,000+2,000$ e $2,000+2,000 \mathrm{~kg} \mathrm{ha}^{-1}$ de diuron+ prometryne, respectivamente. Controle considerado muito bom, ou seja, entre 90 e $95 \%$ de controle, foi alcançado pela mistura de $0,500+$ $1,000 \mathrm{~kg} \mathrm{ha}^{-1}$ de diuron e prometryne, respectivamente. Valores tidos como aceitáveis $(80$ a $85 \%$ ) foram obtidos com as doses de $0,500+$ 0,500 e $0,250+0,500 \mathrm{~kg} \mathrm{ha}^{-1}$ dessa associação.

Conclui-se que, para obter acima de 95\% de controle, foi necessário no mínimo $1,00 \mathrm{~kg} \mathrm{ha}{ }^{-1}$ de diuron e $0,500 \mathrm{~kg} \mathrm{ha}^{-1} \mathrm{de}$ prometryne. Para obter controle superior a $80 \%$, foi necessário no mínimo $0,250+$ $0,500 \mathrm{~kg} \mathrm{ha}^{-1}$ de diuron e prometryne, respectivamente.

Tabela 3 - Massa seca e porcentagem de controle visual de E. heterophylla aos 14, 21 e 28 DAA, proporcionadas pelas misturas de diuron e prometryne

\begin{tabular}{|c|c|c|c|c|c|}
\hline \multirow{2}{*}{ Herbicida } & \multirow{2}{*}{ Dose $\left(\mathrm{kg} \mathrm{ha}^{-1}\right)$} & \multirow{2}{*}{$\begin{array}{c}\begin{array}{c}\text { Massa seca } \\
\text { (g por vaso) }\end{array} \\
28 \text { DAA }\end{array}$} & \multicolumn{3}{|c|}{ Controle visual (\%) } \\
\hline & & & $14 \mathrm{DAA}$ & $21 \mathrm{DAA}$ & $28 \mathrm{DAA}$ \\
\hline Diuron + Prometryne & $(0,125+0,125)$ & 0,99 & 2,50 & 7,50 & 7,50 \\
\hline Diuron + Prometryne & $(0,250+0,250)$ & 0,53 & 13,75 & $51,25(+)$ & $51,25(+)$ \\
\hline Diuron + Prometryne & $(0,500+0,500)$ & 0,17 & $63,75(+)$ & $82,50(+)$ & $82,50(+)$ \\
\hline Diuron + Prometryne & $(1,000+1,000)$ & $0,00(-)$ & $96,75(+)$ & $99,50(+)$ & $99,50(+)$ \\
\hline Diuron + Prometryne & $(0,250+0,125)$ & 0,70 & 13,75 & $43,75(+)$ & $43,75(+)$ \\
\hline Diuron + Prometryne & $(0,500+0,250)$ & 0,40 & $46,25(+)$ & $74,25(+)$ & $74,25(+)$ \\
\hline Diuron + Prometryne & $(1,000+0,500)$ & $0,00(-)$ & $97,5(+)$ & $100,00(+)$ & $100,00(+)$ \\
\hline Diuron + Prometryne & $(2,000+1,000)$ & $0,00(-)$ & $98,25(+)$ & $100,00(+)$ & $100,00(+)$ \\
\hline Diuron + Prometryne & $(0,125+0,250)$ & 0,67 & $41,25(+)$ & $53,00(+)$ & $53,00(+)$ \\
\hline Diuron + Prometryne & $(0,250+0,500)$ & 0,28 & $60,00(+)$ & $80,00(+)$ & $80,00(+)$ \\
\hline Diuron + Prometryne & $(0,500+1,000)$ & 0,13 & $78,75(+)$ & $90,00(+)$ & $90,00(+)$ \\
\hline Diuron + Prometryne & $(1,000+2,000)$ & $0,00(-)$ & $95,75(+)$ & $100,00(+)$ & $100,00(+)$ \\
\hline Diuron + Prometryne & $(2,000+2,000)$ & $0,00(-)$ & $97,25(+)$ & $100,00(+)$ & $100,00(+)$ \\
\hline Testemunha & $(0,00+0,00)$ & 0,68 & 0,00 & 0,00 & 0,00 \\
\hline $\operatorname{DMS}(5 \%)$ & & 0,56 & 29,23 & 30,62 & 30,62 \\
\hline
\end{tabular}

Médias seguidas de (+) são significativamente superiores à testemunha a $5 \%$ de probabilidade pelo teste de Dunett. Médias seguidas de (-) são significativamente inferiores à testemunha a $5 \%$ de probabilidade pelo teste de Dunett. 


\section{Diuron+oxyfluorfen}

Para as misturas de diuron+oxyfluorfen, reduções significativas de massa seca em relação à testemunha foram obtidas nas misturas de 1,000+0,096, 1,000+0,192, 0,500+ $0,096,1,000+0,144,0,250+0,096,0,500+0,192$, $1,000+0,288$ e $2,000+0,288 \mathrm{~kg} \mathrm{ha}^{-1} \mathrm{de}$ diuron+oxyfluorfen (Tabela 4).

Em relação à porcentagem de controle visual aos 28 DAA, controle considerado excelente (>95\%) foi obtido pelas misturas de diuron+oxyfluorfen nas doses de 1,00+0,288 e 2,00+0,288 $\mathrm{kg} \mathrm{ha}^{-1}$. A dose de 1,00+0,096 proporcionou controle muito bom $(92,5 \%)$. Controle considerado aceitável (80 a $85 \%$ ) foi alcançado pelas doses de 1,00+0,192, 1,00+ 0,144 e $0,500+0,192 \mathrm{~kg}$ i.a. ha ${ }^{-1}$ de diuron+ oxyfluorfen, respectivamente (Tabela 4).

Laca-Buendia (1985) relata controle de $78,8 \%$ de Cyperus sp. aos 30 dias com a mistura de $0,800+0,800 \mathrm{~kg} \mathrm{ha}^{-1}$ de cyanazine e diuron em solo com $54 \%$ de argila e 1,75\% de matéria orgânica para a cultura algodoeira.
Portanto, os melhores niveis de controle $(>95 \%)$ foram obtidos com misturas contendo $0,288 \mathrm{~kg} \mathrm{ha}^{-1}$ de oxyfluorfen, com 1,000 a $2,000 \mathrm{~kg} \mathrm{ha}^{-1}$ de diuron. Controles entre $80 \mathrm{e}$ $95 \%$ foram obtidos com misturas contendo $1,000 \mathrm{~kg} \mathrm{ha}^{-1}$ de diuron e doses a partir de $0,096 \mathrm{~kg} \mathrm{ha}^{-1}$ de oxyfluorfen. Doses menores de diuron $\left(0,500 \mathrm{~kg}\right.$ ha $\left.{ }^{1}\right)$ só atingiram este nivel de controle quando em mistura com $0,192 \mathrm{~kg} \mathrm{ha}^{-1}$ de oxyfluorfen.

\section{Prometryne + oxyfluorfen}

Para os herbicidas utilizados nesta associação, pode-se observar que reduções significativas de massa seca em relação à testemunha foram obtidas nas doses de $0,500+0,048,1,000+0,096,0,500+0,096$, $1,000+0,144,0,250+0,096,0,500+0,192$, $1,000+0,288,0,500+0,096,1,000+0,192$ e $2,000+0,288 \mathrm{~kg} \mathrm{ha}^{-1}$ de prometryne+ oxyfluorfen (Tabela 5). Nota-se que, mesmo havendo redução significativa de massa seca em relação a testemunha, nenhum dos tratamentos contendo essas misturas

Tabela 4 - Massa seca e porcentagem de controle visual de E. heterophylla aos 14, 21 e 28 DAA, proporcionadas pelas misturas de diuron e oxyfluorfen

\begin{tabular}{|c|c|c|c|c|c|}
\hline \multirow[t]{2}{*}{ Herbicida } & \multirow[t]{2}{*}{ Dose $\left(\mathrm{kg} \mathrm{ha}^{-1}\right)$} & \multirow{2}{*}{$\begin{array}{c}\begin{array}{c}\text { Massa seca } \\
\text { (g por vaso) }\end{array} \\
28 \text { DAA }\end{array}$} & \multicolumn{3}{|c|}{ Controle visual (\%) } \\
\hline & & & $14 \mathrm{DAA}$ & $21 \mathrm{DAA}$ & $28 \mathrm{DAA}$ \\
\hline Diuron + Oxyfluorfen & $(0,250+0,024)$ & 1,03 & 8,75 & 12,50 & 12,50 \\
\hline Diuron + Oxyfluorfen & $(0,500+0,048)$ & 0,47 & $48,75(+)$ & $53,25(+)$ & $53,25(+)$ \\
\hline Diuron + Oxyfluorfen & $(1,000+0,096)$ & $0,09(-)$ & $82,25(+)$ & $92,50(+)$ & $92,50(+)$ \\
\hline Diuron + Oxyfluorfen & $(1,000+0,192)$ & $0,15(-)$ & $80,00(+)$ & $80,50(+)$ & $80,50(+)$ \\
\hline Diuron + Oxyfluorfen & $(0,125+0,024)$ & 0,87 & $32,50(+)$ & 15,00 & 15,00 \\
\hline Diuron + Oxyfluorfen & $(0,250+0,048)$ & 0,82 & 17,50 & 12,50 & 12,50 \\
\hline Diuron + Oxyfluorfen & $(0,500+0,096)$ & $0,27(-)$ & $62,50(+)$ & $72,00(+)$ & $72,00(+)$ \\
\hline Diuron + Oxyfluorfen & $(1,000+0,144)$ & $0,18(-)$ & $63,75(+)$ & $80,25(+)$ & $80,25(+)$ \\
\hline Diuron + Oxyfluorfen & $(0,125+0,048)$ & 0,96 & 27,50 & 20,00 & 20,00 \\
\hline Diuron + Oxyfluorfen & $(0,250+0,096)$ & $0,32(-)$ & $47,50(+)$ & $69,50(+)$ & $69,50(+)$ \\
\hline Diuron + Oxyfluorfen & $(0,500+0,192)$ & $0,12(-)$ & $77,50(+)$ & $84,25(+)$ & $84,25(+)$ \\
\hline Diuron + Oxyfluorfen & $(1,000+0,288)$ & $0,00(-)$ & $98,00(+)$ & $99,00(+)$ & $99,00(+)$ \\
\hline Diuron + Oxyfluorfen & $(0,250+0,048)$ & 0,62 & 13,75 & 28,75 & 28,75 \\
\hline Diuron + Oxyfluorfen & $(2,000+0,288)$ & $0,00(-)$ & $97,00(+)$ & $99,75(+)$ & $99,75(+)$ \\
\hline Testemunha & $(0,00+0,00)$ & 0,92 & 0,00 & 0,00 & 0,00 \\
\hline $\operatorname{DMS}(5 \%)$ & & 0,52 & 29,74 & 31,44 & 31,44 \\
\hline
\end{tabular}

Médias seguidas de (+) são significativamente superiores à testemunha a 5\% de probabilidade pelo teste de Dunett. Médias seguidas de (-) são significativamente inferiores à testemunha a $5 \%$ de probabilidade pelo teste de Dunett. 
promoveu $100 \%$ de redução de massa de E. heterophylla.

Em relação à porcentagem de controle visual proporcionado pelas associações de prometryne+oxyfluorfen (Tabela 5), a única combinação de dose que proporcionou excelente controle aos 28 DAA (>95\%) foi de $1,000+0,288 \mathrm{~kg} \mathrm{ha}^{-1}$. Controle considerado muito bom (90 a 95\%) foi alcançado pela mistura de $2,000+0,288 \mathrm{~kg} \mathrm{ha}^{-1}$. Controle considerado aceitável (80 à $85 \%$ ) foi proporcionado pela mistura de $1,000+0,192 \mathrm{~kg} \mathrm{ha}^{-1}$ aos 28 DAA (Tabela 5).

Yadav et al. (2006), testando os herbicidas prometryne, trifluralin, pendimethalin e oxyfluorfen isoladamente ou em combinação para o controle de plantas daninhas em algodão, concluíram que oxyfluorfen a 0,100 e $0,150 \mathrm{~kg} \mathrm{ha}^{-1}$, aplicado em pré-emergência, além de apresentar controle insuficiente das plantas daninhas $(<40 \%)$, também causou 10 a 20\% de toxicidade ao algodão até os 15 DAA, porém recuperando-se gradualmente mais tarde. Prometryne a $1,5 \mathrm{~kg} \mathrm{ha}^{-1}$ aplicado em mistura com trifluralin a $0,100 \mathrm{~kg} \mathrm{ha}^{-1}$ ou pendimethalin a $1,5 \mathrm{~kg} \mathrm{ha}^{-1}$ em pré-emergência proporcionaram controle de $76-84 \%$ e $71-82 \%$ de Echinochloa colonum e Trianthema portulacastrum, respectivamente.

Em resumo, apenas as misturas contendo $0,288 \mathrm{~kg} \mathrm{ha}^{-1}$ de oxyfluorfen com 1,000 a $2,000 \mathrm{~kg} \mathrm{ha}^{-1}$ de prometryne proporcionaram controle acima de $93 \%$. Com oxyfluorfen a $0,192 \mathrm{~kg} \mathrm{ha}^{-1}$ em mistura com prometryne a 0,500 a $1,000 \mathrm{~kg} \mathrm{ha}^{-1}$ foram obtidos 78 a $81 \%$ de controle de Euphorbia heterophylla, nas condições em que foi realizado este trabalho.

Comparando as três misturas de herbicidas avaliadas neste trabalho, oxyfluorfen+ prometryne foi a associação que resultou em menor número de combinações que promoveram controle aceitável de $E$. heterophylla.

Os herbicidas diuron e prometryne inibidores do fotossistema II - mostraram-se eficientes para o controle de E. heterophylla. Dessa forma, prometryne e diuron poderiam substituir os herbicidas inibidores da ALS em áreas com problemas de infestação por E. heterophylla resistente a esses herbicidas, apresentando-se, portanto, como opções para o manejo dessa espécie na cultura do algodão.

Da mesma forma, E. heterophylla mostrouse sensivel também ao herbicida oxyfluorfen, cujo mecanismo de ação é a inibição da enzima protoporfirinogênio oxidase (PROTOX). Esse fato sugere que, em áreas com alta infestação de leiteiro, a rotação de mecanismos de ação utilizando oxyfluorfen pode ser uma opção eficiente no controle de E. heterophylla, além de contribuir no controle de plantas tolerantes ou resistentes selecionadas pela aplicação repetitiva de mecanismos de ação comumente utilizados.

De modo geral, nenhuma das misturas contendo oxyfluorfen promoveu ganhos de controle em relação aos resultados esperados para a aplicação isolada dos herbicidas avaliados (Tabela 6). De acordo com o modelo de cálculo dos efeitos de misturas proposto por Colby (1967), os efeitos mais evidentes de antagonismo para as misturas contendo oxyfluorfen foram observados quando a dose de $0,192 \mathrm{~kg} \mathrm{ha}^{-1}$ desse herbicida foi misturada à dose de $1,000 \mathrm{~kg} \mathrm{ha}^{-1}$ de diuron e de prometryne (Tabela 6). Misturas de diuron e prometryne apresentaram, no minimo, efeito aditivo, isto é, o uso simultâneo desses herbicidas em mistura em tanque não alterou o desempenho dessas moléculas.

A aparente ambiguidade de resultados sugere, na verdade, que as respostas em termos de sinergismo ou antagonismo para uma determinada mistura podem diferir em relação à infestação, às doses utilizadas ou aos herbicidas envolvidos.

Desse modo, quando se optar pela utilização de misturas de herbicidas, deve-se analisar antes da escolha, visto que elas devem apresentar algumas vantagens em relação à aplicação dos produtos isolados. A mistura deve proporcionar controle total no mínimo igual (aditivo) ou maior (sinergismo) em relação aos produtos quando aplicados isoladamente, a fim de que possa ser considerada de interesse agronômico.

Assim, para as condições avaliadas e dentro da faixa de doses estudadas, diuron, oxyfluorfen e prometryne, aplicados isoladamente, mostraram-se eficientes no controle de Euphorbia heterophylla. As misturas com 
Tabela 5 - Massa seca e porcentagem de controle visual de E. heterophylla aos 14, 21 e 28 DAA, proporcionadas pelas misturas de prometryne e oxyfluorfen

\begin{tabular}{|l|c|c|c|c|c|}
\hline \multirow{2}{*}{ Herbicida } & \multirow{2}{*}{ Dose $\left(\mathrm{kg} \mathrm{ha}^{-1}\right)$} & \multicolumn{2}{c|}{$\begin{array}{c}\text { Massa seca } \\
\text { (g por vaso) }\end{array}$} & \multicolumn{2}{c|}{ Controle visual (\%) } \\
\cline { 3 - 6 } & & 28 DAA & 14 DAA & 21 DAA & 28 DAA \\
\hline Prometryne + Oxyfluorfen & $(0,250+0,024)$ & 0,79 & $31,25(+)$ & $35,00(+)$ & $35,00(+)$ \\
\hline Prometryne + Oxyfluorfen & $(0,500+0,048)$ & $0,40(-)$ & $45,00(+)$ & $56,25(+)$ & $56,25(+)$ \\
\hline Prometryne + Oxyfluorfen & $(1,000+0,096)$ & $0,23(-)$ & $68,75(+)$ & $73,25(+)$ & $73,25(+)$ \\
\hline Prometryne + Oxyfluorfen & $(0,125+0,024)$ & 0,83 & 16,25 & 2,50 & 2,50 \\
\hline Prometryne + Oxyfluorfen & $(0,250+0,048)$ & 0,77 & $23,75(+)$ & 15,00 & 15,00 \\
\hline Prometryne + Oxyfluorfen & $(0,500+0,096)$ & $0,41(-)$ & $58,75(+)$ & $50,00(+)$ & $50,00(+)$ \\
\hline Prometryne + Oxyfluorfen & $(1,000+0,144)$ & $0,25(-)$ & $77,50(+)$ & $64,75(+)$ & $64,75(+)$ \\
\hline Prometryne + Oxyfluorfen & $(0,125+0,048)$ & 0,81 & $30,00(+)$ & 6,25 & 6,25 \\
\hline Prometryne + Oxyfluorfen & $(0,250+0,096)$ & $0,35(-)$ & $60,00(+)$ & $58,75(+)$ & $58,75(+)$ \\
\hline Prometryne + Oxyfluorfen & $(0,500+0,192)$ & $0,16(-)$ & $80,00(+)$ & $78,25(+)$ & $78,25(+)$ \\
\hline Prometryne + Oxyfluorfen & $(1,000+0,288)$ & $0,04(-)$ & $85,00(+)$ & $95,25(+)$ & $95,25(+)$ \\
\hline Prometryne + Oxyfluorfen & $(0,500+0,096)$ & $0,45(-)$ & $50,00(+)$ & $43,75(+)$ & $43,75(+)$ \\
\hline Prometryne + Oxyfluorfen & $(1,000+0,192)$ & $0,12(-)$ & $75,00(+)$ & $81,25(+)$ & $81,25(+)$ \\
\hline Prometryne + Oxyfluorfen & $(2,000+0,288)$ & $0,04(-)$ & $91,00(+)$ & $93,25(+)$ & $93,25(+)$ \\
\hline Testemunha & $(0,00+0,00)$ & 1,15 & 0,00 & 0,00 \\
\hline DMS (5\%) & & 0,50 & 21,99 & 32,39 & 0,00 \\
\hline
\end{tabular}

Médias seguidas de (+) são significativamente superiores à testemunha a 5\% de probabilidade pelo teste de Dunett. Médias seguidas de (-) são significativamente inferiores à testemunha a $5 \%$ de probabilidade pelo teste de Dunett.

Tabela 6 - Porcentagens de controle dos herbicidas isolados, controle esperado, controle observado pelas misturas e o efeito apresentado pelas misturas de diuron, oxyfluorfen e prometryne (Colby, 1967) para Euphorbia heterophylla

\begin{tabular}{|c|c|c|c|c|c|c|}
\hline \multirow{2}{*}{\multicolumn{2}{|c|}{ Dose $\left(\mathrm{kg} \mathrm{ha}^{-1}\right)$}} & \multirow{2}{*}{\multicolumn{2}{|c|}{$\begin{array}{l}\text { Controle proporcionado pela } \\
\text { aplicação isolada de: }\end{array}$}} & \multicolumn{3}{|c|}{ Controle proporcionado pelas misturas } \\
\hline & & & & \multirow[t]{2}{*}{ Esperado } & \multirow[t]{2}{*}{ Observado } & \multirow[t]{2}{*}{ Efeito da mistura } \\
\hline Diuron & Prometryne & Diuron & Prometryne & & & \\
\hline 1,000 & 1,000 & 94,00 & 73,75 & 98,425 & 99,500 & Sinergístico/aditivo \\
\hline 2,000 & 2,000 & 100,00 & 95,75 & 100,000 & 100,000 & Aditivo \\
\hline 1,000 & 2,000 & 94,00 & 95,75 & 99,745 & 100,000 & Sinergístico/aditivo \\
\hline 2,000 & 1,000 & 100,00 & 73,75 & 100,000 & 100,000 & Aditivo \\
\hline Diuron & Oxyfluorfen & Diuron & Oxyfluorfen & & & \\
\hline 1,000 & 0,192 & 94,00 & 83,75 & 99,025 & 80,500 & Antagonístico \\
\hline 2,000 & 0,288 & 100,00 & 99,00 & 100,000 & 99,750 & Antagonístico/aditivo \\
\hline 1,000 & 0,288 & 94,00 & 99,00 & 99,940 & 99,000 & Antagonismo/aditivo \\
\hline Prometryne & Oxyfluorfen & Prometryne & Oxyfluorfen & & & \\
\hline 1,000 & 0,192 & 73,75 & 83,75 & 95,730 & 81,250 & Antagonístico \\
\hline 2,000 & 0,288 & 95,75 & 99,00 & 99,960 & 93,250 & Antagonístico/aditivo \\
\hline 1,000 & 0,288 & 73,75 & 99,00 & 99,740 & 95,250 & Antagonístico/aditivo \\
\hline
\end{tabular}

oxyfluorfen apresentaram-se, de modo geral, como aditivas ou antagonísticas, ao passo que misturas de diuron+prometryne foram aditivas ou sinergísticas. Das três misturas avaliadas, oxyfluorfen+prometryne foi a associação que resultou em menor número de combinações que promoveram controle aceitável do leiteiro. 


\section{LITERATURA CITADA}

CARVALHO, S. J. P. et al. Curvas de dose-resposta para avaliação do controle de fluxos de emergência de plantas daninhas pelo herbicida imazapic. Planta Daninha, v. 23, n. 3 , p. $535-542,2005$.

CASTRO, C.; BRIGHENTI, A. M.; OLIVEIRA JR, A. Mistura em tanque de boro e herbicidas em semeadura convencional de girassol. Planta Daninha, v. 20, n. 1, p. $83-91,2002$

COLBY, S. R. Calculating synergistic and antagonistic responses of herbicide combinations. Weeds, v. 15, p. 20-22, 1967.

CRUZ, L. S. P.; TOLEDO, N. M. P. Aplicação pré-emergente de misturas de alachlor com diuron e cymazine para controle de plantas daninhas em algodão IAC 17. Planta Daninha, v. 5 , n. 2, p. 57-61, 1982

DOURADO NETO, D. et al. Controle químico de plantas infestantes em pré e em pós-emergência da cultura de cana-de-açúcar. Revista da FZVA, v. 12, n. 1, p. 14-24. 2005.

LACA-BUENDIA, J. P. Controle de plantas daninhas com cyanazine aplicado e mistura com outros herbicidas, na cultura do algodão (Gossypium hirsutum L.).

Planta Daninha, v. 9, n. 1/2, p. 72-80, 1985.

LACA-BUENDIA, J. P. Controle das plantas daninhas na cultura do algodoeiro. Inf. Agropec., v. 8, n. 92, p. 37-47, 1990.
MONQUEIRO, P. A.; CHRISTOFOLLETI, P. J.; DIAS, C. T. S. Resistência de plantas daninhas aos herbicidas inibidores da ALS na cultura da soja (Glycine max). Planta Daninha, v. 18, n. 3 , p. $419-423,2000$.

MONQUERO, P. A.; SILVA, A. C. Efeito do período de chuva no controle de Euphorbia heterophylla e Ipomoea purpurea pelos herbicidas glyphosate e sulfosate Planta Daninha, v. 25, n. 2, p. 399-404, 2007.

OLIVEIRA, M. F. Comportamento de herbicidas no ambiente. In: OLIVEIRA JR., R. S.; CONSTANTIN, J. (Eds.). Plantas daninhas e seu manejo. Guaíba: Agropecuária, 2001. p. 315-362.

PRATA, F.; LAVORENTI, A. Comportamento de herbicidas no solo: influência da matéria orgânica. R. Bioci., v. 6, n. 2, p. $17-22,2000$

RODRIGUES, B. N.; ALMEIDA, F. S. Guia de herbicidas. 5.ed. Londrina: 1998. 592 p.

SAEG - Sistema para Análises Estatísticas, versão 7.0, Viçosa, MG: Fundação Arthur Bernardes, 1997.

SEEFELDT, S. S.; JENSEN, S. E.; FUERST, E. P. Loglogistic analysis of herbicide dose-response relationship. Weed Technol., v. 9, p. 218-227, 1995.

VARGAS, L. et al. Identificação e manejo de plantas daninhas resistentes a herbicidas. Viçosa, MG: Jard, 1999 $39 \mathrm{p}$.

YADAV, A. et al. Integrated control of weeds in cotton. Environ. Ecol., v. 24S, p. 883-885, 2006. 